\title{
Prediction of the behaviour of CFRPs against high-velocity impact of solids employing an artificial neural network methodology
}

\author{
D. Fernández-Fdz, J. López-Puente *, R. Zaera \\ Department of Continuum Mechanics and Structural Analysis, University Carlos III of Madrid. Avda. de la Universidad, 30, 28911 Leganés, Madrid, Spain
}

\begin{abstract}
A B S T R A C T
A new methodology based on artificial neural networks has been developed to study the high velocity oblique impact of spheres into CFRP laminates. One multilayer perceptron (MLP) is employed to predict the occurrence of perforation of the laminate and a second MLP predicts the residual velocity, the obliq uity of trajectory of the sphere after perforation and the damage extension in the laminate. In order to train and test the networks, multiple impact cases have been generated by finite element numerical sim ulation covering different impact angles and impact velocities of the sphere for a given system sphere/ laminate.
\end{abstract}

\section{Introduction}

Fiber reinforced composite (FRP) materials are used increas ingly in many applications due to their high strength and stiff ness, high resistance to corrosion and fatigue, and low density. The aviation and space industries make extensive use of these materials, since any slight reduction of the total mass of the struc ture means a saving of power and of fuel. The impact between any of those structural materials and solids is inevitable and crit ical. Hail ice, birds, debris, and fragments from tyres or turbine blades may strike the composite laminate at high velocities. In most of these cases, the impact is more likely to be oblique rather than purely normal. FRPs are well known to be extremely vulner able to damage from foreign objects due to the brittleness of the polymeric phase and the poor translaminar properties. The resid ual strength of the laminate decreases and the risk of structural failure under service rises. As a result the threat of impact is a significant design consideration and foreign object damage must be predictable.

The traditional method to analyse and design FRPs that may re ceive high velocity impacts during its service life is the empirical one, which consists of performing real test to composite specimens [1 3 3]. This methodology is costly as testing under this high speed conditions requires sophisticated facilities and equipment. It be comes even more costly when the aim of the test goes beyond the discovery of the resistance quality of the target; to gather infor

* Corresponding author. Tel.: +34 916248881; fax: +34 916249973.

E-mail address: jlpuente@ing.uc3m.es (J. López-Puente). mation on the deformation of the target or on the position of the projectile during penetration, ultra rapid cameras are required. An increasingly frequent trend to upgrade cost efficiency is to re duce the experimental testing by using analytical or numerical simulation. Numerical codes solve the variational equations of the Mechanics of Continuous Media by means of finite element or finite difference methods. Some works on numerical simulation of ballistic impacts in FRPs appear in [4 6]. Although less expen sive than experimental testing, they also entail a high cost because of the price of the codes and the long computational time. For a quick prediction of the performance of the laminate, analytical models have been also proposed $\left[\begin{array}{ll}7 & 10\end{array}\right]$. These are derived from a qualitative understanding of the macroscopic phenomena. The main drawback of this methodology is the limited applicability of a given model to a short range of impact conditions (type of FRP and projectile, impact regime).

Consequently, a design engineer needs a low cost tool that would enable an impact problem to be solved in the shortest pos sible time, that would be easy to use, and that would give the re quired precision. This would permit the simulation of a large number of impact problems in the early design stages. In the mul tidisciplinary framework in which engineering is being developed nowadays, and with the huge advance in artificial computation techniques, artificial neural networks (ANN) are providing fast re sults to mechanical problems. Their advantages become manifest when the problem is characterized by a high nonlinearity, as it oc curs in the case of high velocity impact on FRPs laminates. In the field of composite materials, numerous authors have developed techniques based on ANN, coupled with FEM numerical simulation or experimental approaches. Although most of the works 
published in this field are related to damage detection [11 15], some papers deal with the analysis of static, crushing, dynamic, creep or tribological behaviour of composites, using different ANNs (see the work due to Mahdi and El Kadi [16] or the complete re view presented by El Kadi [17]). Focusing on the field of high speed impact, a few works using ANNs can be found: Chandrashekhara et al. [18] have studied the contact force for low velocity impacts on laminated composite plates and Fernandez Fdz et al. [19] have predicted the ballistic behaviour of composite ceramic metallic ar mors against high velocity impact of solids.

This work presents the results from using an ANN as an alterna tive to classical methods in the prediction of the performance of thin CFRP woven laminates against high speed oblique impacts of spheres. In a first phase, a number of impact cases are randomly generated, varying the values of the parameters which define the impact problem. After simulation of each case using a finite ele ment code (validated with experimental results), the above men tioned parameters and the results of the simulation (residual velocity, obliquity of trajectory of the projectile after perforation, damage extent in the laminate) are used respectively as input and output data to train and validate the neural network. The re sults of the ANN are reliable and the tool shows a great handling simplicity as well as low computational cost.

\section{Neural network approach}

\subsection{Architecture of the multilayer perceptron}

Multilayer perceptron (MLP) is the ANN most commonly ap plied in Mechanics although other topologies such as radial basis function network (RBFN) have been successfully used in this field $[20,21]$. The massively parallel structure of ANNs can be under stood from the behaviour of one simple processing element or arti ficial neuron. In feedforward networks such as the MLP, an artificial neuron (Fig. 1a) receives signals $x_{j}$ from the previous neurons in the network structure; these signals are multiplied by synapse weights $\varphi_{j i}$. The neuron is activated if the sum of the weighted sig nals is greater than an activation threshold $b_{i}$. In this case the neu ron output $v_{i}$ will be determined by the expression

$v_{i} \quad f_{i}\left(\begin{array}{ll}\sum_{j 1}^{K} \varphi_{j i} x_{j} & b_{i}\end{array}\right)$

being $f_{i}$ the activation function. Most common functions are logistic, hyperbolic tangent, linear and threshold functions (Fig. 1b). The choice of $f_{i}$ is made according to the problem to be solved (different functions must be trained) and according to the kind of layer where the neuron is to work (input, hidden or output layer) [22].

The architecture of a MLP (Fig. 2) is characterized by grouping neurons in the input, hidden and output layers. Connections are made from the input to the output layer, mapping a $\mathrm{N}$ component vector $\mathbf{x}$ containing the input variables in a M component vector $\mathbf{y}$ containing the output variables, so that a MLP defines a nonlinear continuous function $\mathscr{F}$ from $R^{\mathrm{N}}$ to $R^{\mathrm{M}}$

y $\mathscr{F}(\mathbf{x}, \Phi, \beta)$

$\Phi$ and $\beta$ being the set of weights and thresholds. These values must be adjusted during the training process to minimize the error resulting from the MLP when predicting an output $\mathbf{y}$ corresponding to an input $\mathbf{x}$.

\subsection{Training algorithm}

The patterns $(\mathbf{x}, \mathbf{y})_{s}$ form the variability space $\mathscr{R}$ of the excitation and response of the studied system (Fig. 3a). During the training process the MLP derives the characteristics of the system from a re duced set of training patterns $\mathscr{K} \quad\left\{(\mathbf{x}, \mathbf{y})_{s}^{\mathscr{K}} \mid s \quad 1, \ldots, K\right\} \subset \mathscr{R}$ in which both inputs and outputs are known, which is randomly divided in the subset of learning $\mathscr{L} \quad\left\{(\mathbf{x}, \mathbf{y})_{s}^{\mathscr{L}} \mid s \quad 1, \ldots, L\right\} \subset \mathscr{K}$ and the subset of cross validation $\mathscr{V} \quad\left\{(\mathbf{x}, \mathbf{y})_{s}^{\mathscr{V}} \mid s \quad 1, \ldots, V\right\} \subset \mathscr{K}$. The first set is used to calculate the values of $\Phi$ and $\beta$ through an

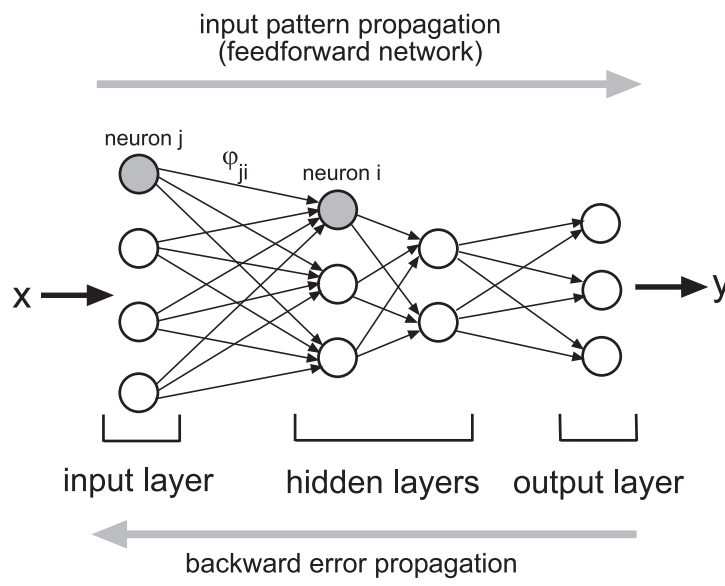

Fig. 2. Architecture of a multilayer perceptron: feedforward network with backward propagation error. Two hidden layers (4-3-2-3).
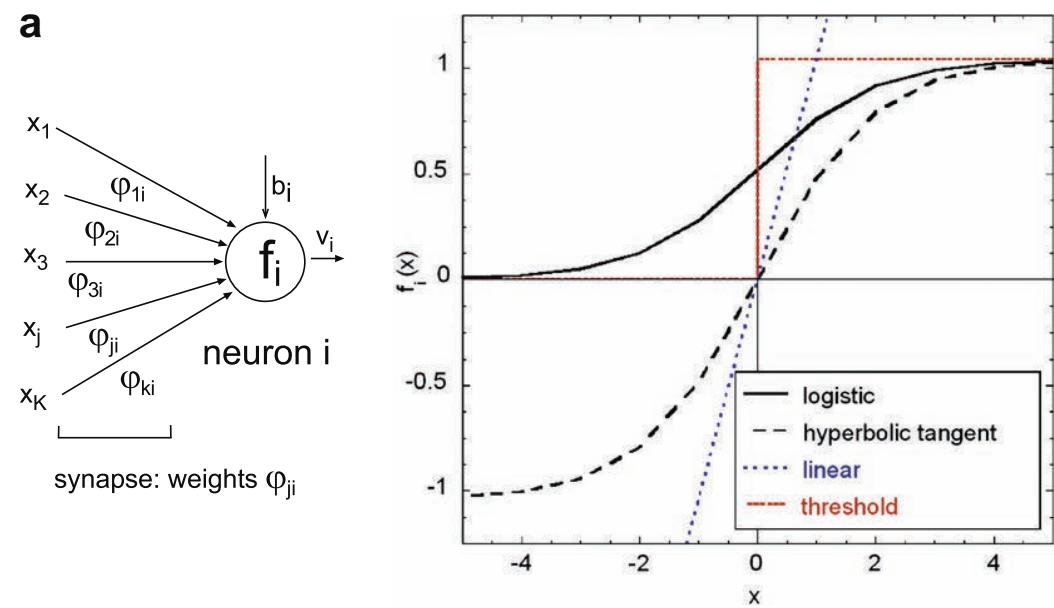

Fig. 1. (a) Processing element: artificial neuron. (b) Most common activation functions. 
a

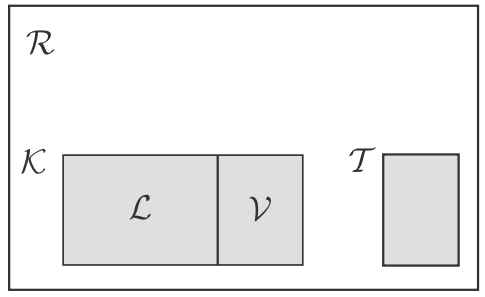

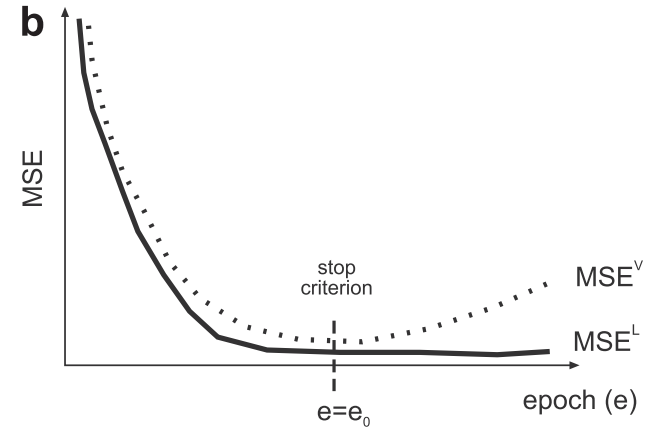

Fig. 3. (a) Variability space $\mathscr{R}$ of $(\mathbf{x}, \mathbf{y})$ : sets of patterns used in training $(\mathscr{K})$ and testing $(\mathscr{T})$ the network. (b) Stop criterion employed in the training algorithm: MSE ${ }^{\mathscr{\gamma}}$ vs. iteration number (epoch).

algorithm called Backpropagation (abbreviation of "Backwards propagation of the errors"). The algorithm iteratively modifies the initial values of weights and thresholds to arrive at the mini mum value of a function which measures the predictive error of the network, following the direction of the gradient descent of this function. Although there are many definitions of the error, the most common is the mean squared one

MSE $\frac{1}{P} \sum_{s}^{P} \mathbf{e}(s)$

$\mathbf{e}(s)$ being the squared error for a pattern $s$

$\mathbf{e}(s) \quad \frac{1}{2} \sum_{i=1}^{M}\left(y_{i}^{*}(s) \quad y_{i}(s)\right)^{2}$

where $y_{i}^{*}(s)$ and $y_{i}(s)$ are respectively the desired and predicted out puts for parameter $i$ in the output pattern $s$ (of dimension $M$ ). A commonly adopted procedure to correct the values of $\Phi$ and $\beta$ is the stochastic gradient descent, which uses the error of a pattern $\mathbf{e}(s)$, instead of a global measure of the error, according to the expressions

$$
\begin{array}{lll}
\varphi_{j i}(s+1) & \varphi_{j i}(s) & \xi \frac{\partial \mathbf{e}(s)}{\partial \varphi_{j i}} \\
b_{i}(s+1) & b_{i}(s) & \xi \frac{\partial \mathbf{e}(s)}{\partial b_{i}}
\end{array}
$$

$\xi$ being the learning rate. The training process can be summarized as follows:

- Weights and thresholds are randomly initialized.

- The input $\mathbf{x}^{\mathscr{L}}(1)$ (from first learning pattern) is propagated, an output $\mathbf{y}(1)$ is determined and error $\mathbf{e}(1)$ is computed.

- The Backpropagation algorithm is applied and weights and thresholds are incremented in negative direction of the error gradient.

- The two previous steps are repeated for the rest of the learning patterns $(\mathbf{x}, \mathbf{y})_{s}^{\mathscr{L}}$, updating $\Phi$ and $\beta$ each time.

- The global learning error $\mathbf{M S E}^{\mathscr{L}}$ is computed completing an epoch (learning cycle).

- Consecutive epochs are repeated until a stable value of $\mathbf{M S E}^{\mathscr{L}}$ is reached (Fig. 3b)

The cross validation subset $\mathscr{V}$ of training patterns is used dur ing the application of the Backpropagation algorithm to prevent overlearning of the MLP. This spurious effect consists in an accurate prediction of the output belonging to the patterns of the learning subset $\mathscr{L}$ but not for independent inputs. Thus, the cross valida tion global error $\mathbf{M S E}^{\mathscr{\gamma}}$ is obtained at the end of each epoch and the learning algorithm is stopped when this error starts to increase (Fig. 3b). Once the training algorithm has finished, the testing set
$\mathscr{T}\left\{(\mathbf{x}, \mathbf{y})_{s}^{\mathscr{T}} \mid S \quad 1, \ldots, T\right\} \subset \mathscr{R}$ is used to test the predictive ability of the MLP with patterns independent from those employed during training.

\section{Data generation from numerical simulations}

\subsection{Numerical simulation tool}

A finite element model has been used to generate the data used to train the neural network. With this numerical procedure the high velocity impact of spherical projectiles against carbon fiber epoxy matrix laminates has been simulated. A woven laminate was selected for this study which is widely used in the aeronautical and aerospace industries for panels subjected to torsion or to shear stresses.

The numerical simulations were performed using the code ABA QUS/Explicit [23]. The material model used in this work is a mod ification of that proposed by Hou et al. [24], which was developed for tape plies; hence some modifications were necessary due to this different laminate architecture. In particular, only two damage mechanisms were considered, but with three damage parameters (two related to fiber failure and one to delamination). This damage parameter could adopt a value between 0 and 1 ; when its value reaches 1 , some of the stress components are set to zero, simulat ing the lack of resistance due to breakage.

Fiber failure: this failure mechanism must be extended in both in plane directions, to model possible breakage in felt and warp fi bers. The two equations describing this failure are:

$d_{f 1}\left(\frac{\sigma_{11}}{X_{\mathrm{T}}}\right)^{2}+\left(\frac{\sigma_{12}^{2}+\sigma_{13}^{2}}{S_{f}^{2}}\right) \geqslant 1$
$d_{f 2}\left(\frac{\sigma_{22}}{X_{\mathrm{T}}}\right)^{2}+\left(\frac{\sigma_{12}^{2}+\sigma_{23}^{2}}{S_{f}^{2}}\right) \geqslant 1$

the constants $X_{\mathrm{T}}$ and $S_{f}$ are the tensile strength and the shear strength in the fiber direction respectively $[4,25]$. On fiber failure, some coefficients of the stiffness tensor are set to zero so that the stress components appearing in the corresponding equation are null at the integration point.

Delamination: since the interlaminar surface is the weakest one in a woven laminate, delamination failure needs to be considered. The delamination failure criteria remains the same way as in the Hou et al. model [24] for laminates made with tape plies. The equa tion for this breakage mechanism is:

$d_{d}\left(\frac{\sigma_{33}}{Z_{\mathrm{T}}}\right)^{2}+\left(\frac{\sigma_{23}}{S_{23}}\right)^{2}+\left(\frac{\sigma_{13}}{S_{23}}\right)^{2} \geqslant 1$ with $\sigma_{33} \geqslant 0$

in which $Z_{\mathrm{T}}$ is the tensile strength in the through thickness direc tion, and $S_{23}$ is the shear strength in the transverse and through thickness plane. When $d_{d} \quad 1, \sigma_{33}, \sigma_{13}$ and $\sigma_{23}$ are set to zero. 
To avoid mesh distortion an element removal criterion was de fined. At each time increment, the longitudinal strain is evaluated in each fiber direction of the ply; if one of these reaches an ultimate value, the element is removed.

\subsection{Model validation}

In order to validate the above mentioned numerical model, experimental tests were conducted. The laminate was provided by SACESA (Spain) from woven AS4/3552 prepegs manufactured by HEXCEL, with a volumetric fibre content of $60 \%$. The stacking se quence was $[0]_{10}$, with a total thickness of $2.2 \mathrm{~mm}$. The elastic mod uli and strength properties are given in Table 1 . The specimen size was $80 \times 80 \mathrm{~mm}^{2}$.

For the impact tests a tempered steel projectile of $1.73 \mathrm{~g}$ mass was used; its shape was spherical to avoid scattered results due to changes in the yaw angle. The projectile material was hard en ough to behave elastically during impact. A SABRE gas gun (Fig. 4) was used to launch the spherical projectiles. It uses helium gas at a pressure of 300 bar to impel the fragment at velocities up to $550 \mathrm{~m} / \mathrm{s}$. This experimental device has two photoelectric cells that detect the passage of the projectile, from which the impact velocity is determined.

For the validation of the numerical model, two variables were selected: residual velocity of the projectile and damaged extent of the laminate. In case of perforation, the residual velocity was

Table 1

Material ply properties and critical values of the strain for element deletion, provided by the manufacturer

\begin{tabular}{ll}
\hline Property & Value \\
\hline Resin content \% & 40 \\
$E_{1}(\mathrm{GPa})$ & 68.5 \\
$E_{2}(\mathrm{GPa})$ & 68.5 \\
$G_{12}(\mathrm{GPa})$ & 3.7 \\
$v_{12}$ & 0.11 \\
$X_{t}(\mathrm{MPa})$ & 795 \\
$X_{c}(\mathrm{MPa})$ & 860 \\
$Y_{t}(\mathrm{MPa})$ & 795 \\
$Y_{c}(\mathrm{MPa})$ & 860 \\
$S_{t}(\mathrm{MPa})$ & 98 \\
$Z_{\mathrm{T}}(\mathrm{MPa})$ & 55 \\
$S_{23}(\mathrm{MPa})$ & 64 \\
$\varepsilon_{1 c}$ & 0.02 \\
$\varepsilon_{2 c}$ & 0.02 \\
$\varepsilon_{3 c}$ & 0.03 \\
\hline
\end{tabular}

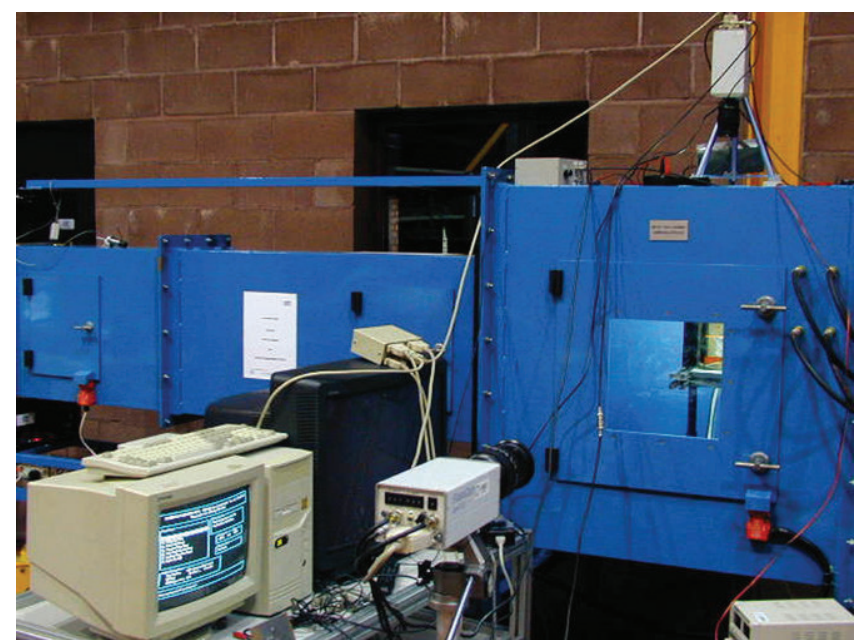

Fig. 4. Gas gun used in the experimental test. measured by high speed cameras placed beside the impact cham ber to photograph the projectile after perforation. Knowing the frame rate and the displacement of the projectile between two frames, the residual velocity was immediately calculated. For the measurement of the damaged extent, the specimens were analysed using the non destructive technique $\mathrm{C}$ scan, which draws a clear map of the damaged zone; the damage was quantified by treating these maps with an image processing software. Tests were made at velocities of between 60 and $550 \mathrm{~m} / \mathrm{s}$, at two different impact angles, $0^{\circ}$ and $45^{\circ}$. Comparisons of experimental test and numeri cal results gave very good correlations $[4,10,25]$.

\subsection{Impact cases generation}

Different cases were simulated to create the data for the neural network. The simulations covered a wide range of velocities, be tween 60 and $550 \mathrm{~m} / \mathrm{s}$, and 4 different impact angles: $0^{\circ}, 30^{\circ}, 45^{\circ}$ and $60^{\circ}$. A total number of 65 cases (defined by an impact angle and velocity) were simulated. Table 2 shows the impact velocities simulated for each impact angle; the increment in the impact velocity is smaller for values close to the ballistic limit, in order to obtain a more precise value of this parameter. Fig. 5a shows the numerical values of the residual velocity $V_{\mathrm{r}}$ as a function of the impact velocity $V_{\mathrm{i}}$ for the different impact angles considered. It's worth to note that at high impact velocities, the four curves converge. Since inertial effects are prevalent at high velocities and the cylindrical volume of laminate (plug) pushed by the pro jectile is independent of the obliquity angle $[10,25]$, no differences are observed in these curves. This can be also noticed by plotting the relation between the normal and tangential components of ini tial velocity ( $V_{\text {in }}$ and $V_{\text {it }}$ respectively) and the normal and tangen tial components of the residual velocity ( $V_{\mathrm{rn}}$ and $V_{\mathrm{rt}}$ respectively) defined in Fig. 5: at high velocities the curves corresponding to the different impact angles coincide (Figs. 5b and c). As the impact velocity decreases, the relation between normal velocities is still coincident for the different impact angles, even at the ballistic limit $\left(V_{\mathrm{in}} \approx 100 \mathrm{~m} / \mathrm{s}\right)$, since the path covered by the projectile in the normal direction is not affected by the obliquity. However, the curves corresponding to tangent velocities diverge when the initial velocity approaches the ballistic limit (Fig. 5c), since the path cov ered by the projectile in the tangent direction is strongly affected by the obliquity. At low velocities, the work done by the projectile to crush the material starts to play a role [10], and an increase in the length covered by the projectile in the tangent direction leads to a higher decrease in the residual velocity. Consequently, the bal listic limit increases with the obliquity angle $\theta$ (Fig. 5a and c).

Table 2

Set of impact velocities $(\mathrm{m} / \mathrm{s})$ and impact angles considered for numerical simulation

\begin{tabular}{rrrr}
\hline $0^{\circ}$ & $30^{\circ}$ & $45^{\circ}$ & $60^{\circ}$ \\
\hline 62 & 75 & 75 & 75 \\
75 & 88 & 88 & 88 \\
88 & 100 & 100 & 100 \\
100 & 112 & 112 & 112 \\
112 & 125 & 125 & 125 \\
125 & 137 & 137 & 137 \\
137 & 150 & 150 & 150 \\
150 & 162 & 162 & 162 \\
162 & 184.5 & 175 & 175 \\
175 & 200 & 200 & 200 \\
200 & 300 & 250 & 212 \\
300 & 400 & 300 & 225 \\
400 & 450 & 400 & 250 \\
500 & 500 & 450 & 300 \\
600 & 550 & 500 & 400 \\
& 600 & 550 & 500 \\
& & 600 & 600 \\
\hline
\end{tabular}



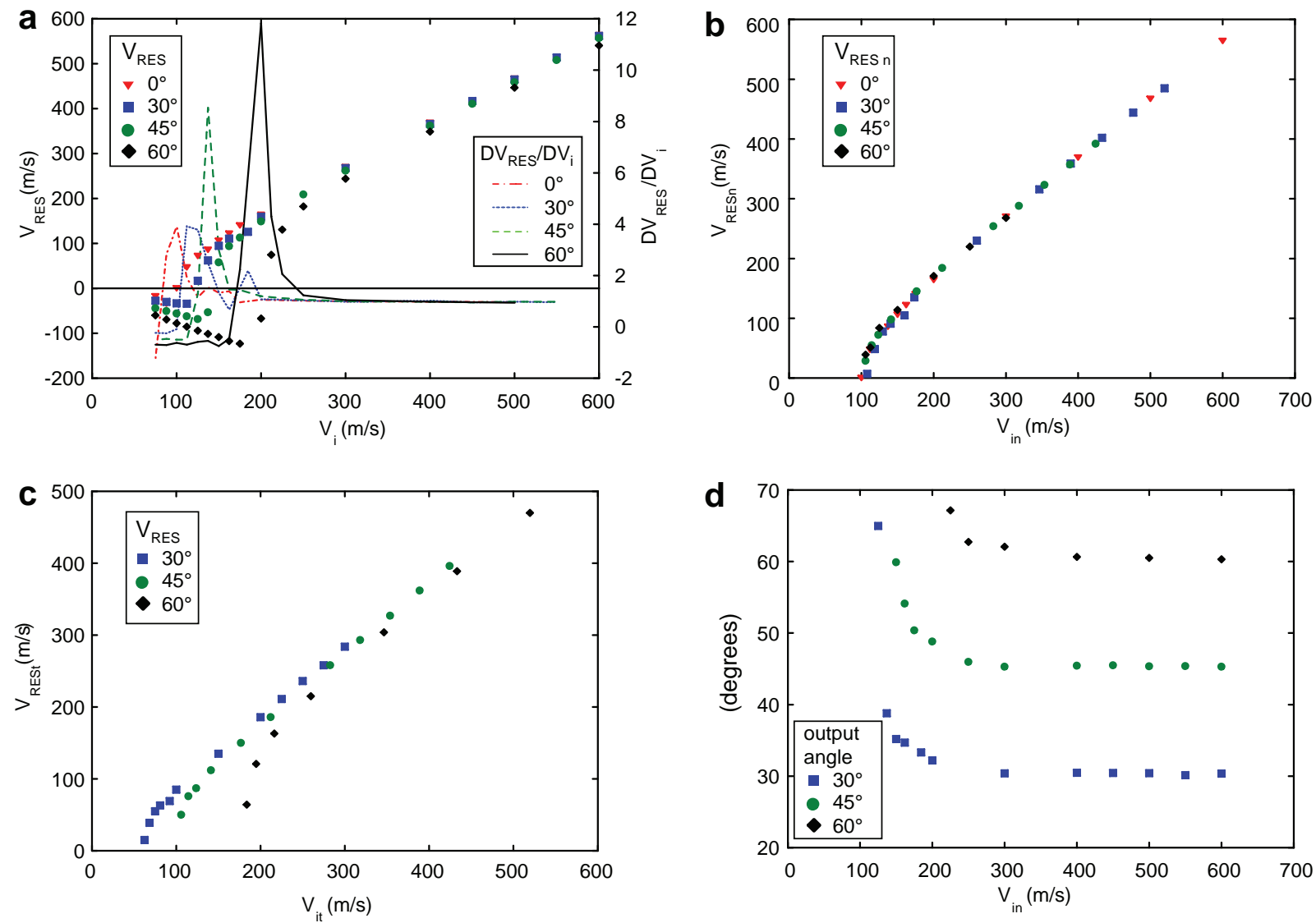

Fig. 5. Numerical results obtained with the model: (a) residual velocity and its derivative vs. impact velocity, (b) normal component of residual velocity vs. normal component of impact velocity, (c) tangent component of residual velocity vs. tangent component of impact velocity and (d) residual angle vs. impact velocity.

Moreover, this effect is amplified by the deviation of the trajec tory suffered by the projectile at velocities close to the ballistic lim it, due to the unbalanced force exerted by the laminate (Fig. 6). As can be seen in Fig. $5 d$, the exit angle $\varphi$ increases sharply as the im pact velocity approaches the ballistic limit.

This also influences the slope of $V_{\mathrm{i}} \quad V_{\mathrm{r}}$ curves at the ballistic limit: the length of the laminate penetrated by the projectile is

$L \quad \frac{h}{\cos \theta}$

$h$ being the thickness of the laminate. Differentiation of $L$

$\delta L \quad \frac{h \operatorname{tg} \theta}{\cos \theta} \delta \theta$

shows how a variation of $L$ with $\theta$ is highly influenced by the value of the obliquity. Then, at high obliquity impact angles, a slight in crease in $V_{\mathrm{i}}$ just above the ballistic limit leads to a large decrease in $\varphi$ and $L$, and to high increase in $V_{\mathrm{r}}$ (Fig. $5 \mathrm{~d}$ ).

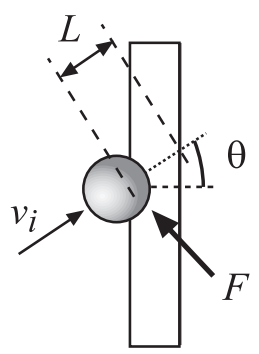

Fig. 6. Unbalanced force exerted by the laminate.

\section{Multilayer Perceptron Development}

Two MLP were developed employing the neural network simu lation code NeuroSolutions for Excel v4.21 [26]. The first one (MLP1) is used to determine the occurrence of the CFRP perfora tion. This involves a classification task, where the network decides whether an impact case, defined by an input pattern, results in the perforation of the laminate or in projectile arrest. The second one (MLP2) solves a regression problem where the network predicts residual velocity, angle of the projectile and damaged extent of the laminate in case of perforation. In both cases, the procedure to develop the network can be summarized as follows:

- Determine the optimum variables that should form the input pattern vector $\mathbf{x}$.

- Randomly select the learning, cross validation and test sets of patterns from the available data generated by numerical simulation.

- Train the network varying the number of neurons in the hidden layer $N_{\mathrm{h}}$ to determine the optimal topology.

- Test the best network obtained during the training process.

\subsection{Input variables}

The input variables that define the problem are the impact velocity $V_{\mathrm{i}}$ and the impact angle $\theta$; the output variables are the damaged area in the laminate $A_{\mathrm{d}}$ and, in the case of perforation, the residual velocity $V_{\mathrm{r}}$ and residual angle $\varphi$. It is not obvious which input variables (or combinations of them) are the best to 
choose in order to improve the predictive ability of the perceptron. For MLP1 the following method to select the input variables, devel oped by the authors, was used to minimize the classification error.

Let $x_{i}$ be the input variable analysed, $\bar{x}_{i}^{\text {perf }}$ and $\hat{x}_{i}^{\text {perf }}$, its mean and standard deviation in the set of patterns corresponding to laminate perforation; $\bar{x}_{i}^{\text {par }}$ and $\hat{x}_{i}^{\text {par }}$, its mean and standard deviation in the set of patterns corresponding to projectile arrest. The relative distance between both means $d_{x i}$ is defined as

$d_{x i} \quad 2 \frac{\left|\bar{x}_{i}^{\text {perf }} \quad \bar{x}_{i}^{\text {par }}\right|}{\bar{x}_{i}^{\text {perf }}+\bar{x}_{i}^{\text {par }}}$

Defining the normalized standard deviation associated with the in put variable $x_{i}$ as

$\hat{\chi}_{i} \frac{\hat{x}_{i}^{\text {perf }}+\hat{\chi}_{i}^{\text {par }}}{\bar{\chi}_{i}^{\text {perf }}+\bar{\chi}_{i}^{\text {par }}}$

One can define an uncertainty parameter $X_{i}$ associated with the in put variable $x_{i}$ dividing Eq. (13) by Eq. (12)

$X_{i} \quad \frac{\hat{x}_{i}}{d_{x i}}$

$X_{i}$ can be used as an estimate of the classification error when a pat tern is classified employing the input variable $x_{i}$. Large values of $X_{i}$ correspond to small distances between means and/or large standard deviations, $x_{i}$ revealing great uncertainty for the prediction of the occurrence of laminate perforation. According to the values shown in Table 3 the two optimum variables $V_{\mathrm{i}}$ and $V_{\text {in }}$ (bold typed in the table) were adopted as input for MLP1.

Table 3

Uncertainty parameters $X_{i}$ to determine optimum input variables (bold typed) for MLP1

\begin{tabular}{ll}
\hline$x_{i}$ & $X_{i}$ \\
\hline $\mathbf{V}_{\mathbf{i}}$ & $\mathbf{0 . 4 9 7}$ \\
$V_{\text {it }}$ & 1.119 \\
$\mathbf{V}_{\text {in }}$ & $\mathbf{0 . 4 5 7}$ \\
$V_{\text {it }} V_{\text {in }}$ & 0.734 \\
$V_{\text {it }} / V_{\text {in }}$ & 1.603 \\
$\theta$ & 1.756
\end{tabular}

Table 4

Values for the correlation coefficient $R_{i j}^{2}$ between input variable $x_{i}$ and output variable $y_{j}$

\begin{tabular}{lllllll}
\hline$\left(x_{i}, y_{j}\right)$ & $V_{\mathrm{r}}$ & $V_{\mathrm{r}} / V_{\mathrm{i}}$ & $V_{\mathrm{rt}}$ & $V_{\mathrm{rn}}$ & $A_{\mathrm{d}}$ & $\varphi$ \\
\hline$V_{i}$ & 0.987 & 0.696 & 0.567 & 0.819 & 0.481 & 0.015 \\
$V_{\text {it }}$ & 0.786 & 0.544 & 0.968 & 0.512 & 0.298 & 0.019 \\
$V_{\text {in }}$ & 0.883 & 0.832 & 0.154 & 0.996 & 0.848 & 0.027 \\
$\theta$ & 0.023 & 0.013 & 0.472 & 0.046 & 0.150 & 0.707 \\
\hline
\end{tabular}

For the determination of the best input variables for the MLP2 a correlation analysis between input and output variables (or combi nations of them) was performed. Table 4 shows the regression coefficient $R_{i j}^{2}$ for the best regression (linear or potential) associated with each combination of variables $\left(x_{i}, y_{j}\right)$. The best global correla tions were made for $V_{\mathrm{i}}$ and $V_{\text {in }}$ as input variables (coinciding with MLP1) and $V_{\mathrm{r}}, V_{\mathrm{rt}}, V_{\mathrm{rn}}$ and $A_{\mathrm{d}}$ as output variables. As a consequence of the low values of regression coefficients associated with the im pact angle $\theta$, this was not considered as an input. It is noteworthy also to note that the angle after impact $\varphi$ can be determined more precisely from the predicted values of $V_{\mathrm{r}}$ and $V_{\mathrm{rn}}$ than from consid ering it as an output variable of the perceptron.

\subsection{Networks training and testing}

Linear functions have been used as activation functions in the input layer, and hyperbolic tangent functions in the hidden and output layers. For the convergence of the training algorithm, a learning rate $\xi 1$ has been used in the hidden layer and $\xi \quad 0.1$ in the output layer. Sixty patterns were employed for the training ( 55 for the learning and 5 for the cross validation) and a set of five patterns, completely foreign to the training data, was employed to test the accuracy of the network. Both networks were trained varying the number of neurons in the hidden layer $N_{\mathrm{h}}$ to determine its optimum value. An initial estimate for $N_{\mathrm{h}}$ can be determined by $N_{\mathrm{h}} \quad \sqrt{ } N M, N$ and $M$ being the number of neurons in the input and output layer respectively [27]. This for mula leads to 2 hidden neurons for the first perceptron and 5 hid den neurons for the second. However, higher values of $N_{\mathrm{h}}$ were also tried: $N_{\mathrm{h} 1} \quad 2,3,4$ for MLP1 and $N_{\mathrm{h} 2} \quad 3,4,5,6$ for MLP2. Table 5 shows the mean squared error obtained for the learning set $\operatorname{MSE}^{\mathscr{L}}$ when the stop criterion is reached (the cross validation er ror $\mathrm{MSE}^{\mathscr{V}}$ is minimum).

The final architecture of both MLPs is shown in Fig. 7. The out put variables for MLP1 are redundant and complementary, that is, if one is true (value 1 ) the other is false (value 0 ). The decomposi tion of a symbolic variable having $p$ categories in $p$ numeric ones is

Table 5

MSE $^{\mathscr{L}}$ for different values of $N_{\mathrm{h}}$ in both MLPs when training algorithm has converged (minimum MSE ${ }^{\mathscr{V}}$ )

\begin{tabular}{|c|c|c|c|}
\hline \multicolumn{2}{|c|}{ MLP1 } & \multicolumn{2}{|c|}{ MLP2 } \\
\hline$N_{\mathrm{h} 1}$ & $\mathrm{MSE}^{\mathscr{L}}$ & $N_{\mathrm{h} 2}$ & $\mathrm{MSE}^{\mathscr{L}}$ \\
\hline 2 & 0.0454 & 3 & 0.0516 \\
\hline 3 & 0.0663 & 4 & 0.0433 \\
\hline \multirow[t]{2}{*}{4} & 0.0712 & 5 & 0.0405 \\
\hline & & 6 & 0.0491 \\
\hline
\end{tabular}

For each MLP, optimum values are typed in bold letters. a

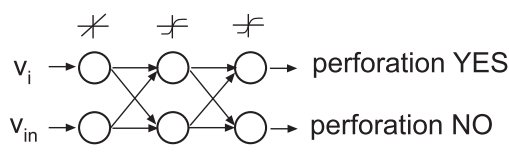

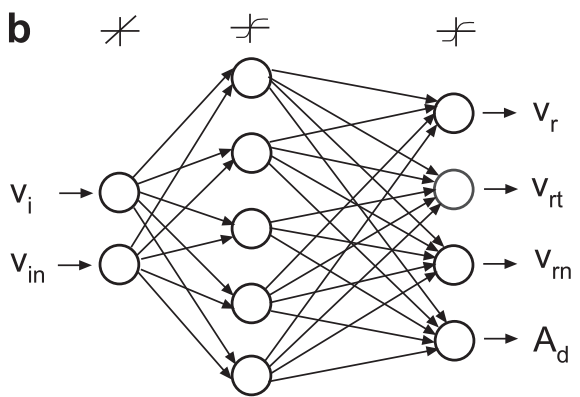

Fig. 7. (a) MLP1 to predict the occurrence of perforation in the laminate. (b) MLP2 to predict damaged area and, in case of perforation, residual velocity. Architectures optimised after training. 
a routine in data preprocessing and reinforces the learning of the network [22]. In this case $p \quad 2$, perforation or arrest.

After training, the MLPs were tested with the independent data of the testing set of patterns. MLP1 has correctly classified all the impact cases and MLP2 has provided an averaged relative error of $E_{V_{\mathrm{r}}} \quad 6.12 \%, E_{\varphi} \quad 5.97 \%$ and $E_{A_{\mathrm{d}}} \quad 4.44 \%$ for residual velocity, residual angle and damaged extent, respectively. This error has been calculated as

$E_{i} \quad 100 \frac{1}{T} \sum_{s=1}^{T} \frac{y_{i}^{\mathscr{T}}(s) y_{i}(s)}{y_{i}^{\mathscr{T}}(s)}$,

where $y_{i}^{\mathscr{T}}(s)$ and $y_{i}(s)$ are respectively the desired and predicted outputs for variable $i$ in the output pattern $s$.

\section{Results and discussion}

Once the MLPs have been tested, they can be employed as a pre dictive tool, obtaining output data (production data) in real time, for impact cases that are defined in the range of variability where the nets have been trained. Although the networks have been

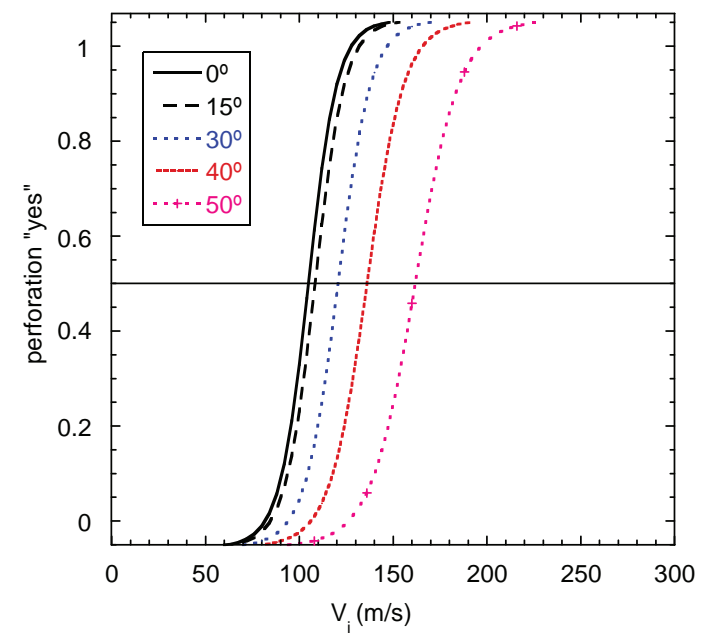

Fig. 8. Values predicted by the network: occurrence of perforation (output network "perforation = yes") vs. impact velocity for five impact angles. trained with data from cases having impact angles of $0^{\circ}, 30^{\circ}, 45^{\circ}$ and $60^{\circ}$, results can be obtained for interpolated angles as is shown in Figs. 8 and 9.

Fig. 8 shows the output of MLP1 "PERFORATION YES" versus the impact velocity for five different impact angles. In the training step this output has been supplied to the net having binary values ( 1 for perforation, 0 for detention) but the MLP obtains continuous values between 0 and 1 . This output may be interpreted as a probability of laminate perforation defined as likeliness, for a pair of data $\left(V_{i}, \theta\right)$ to belong to the laminate perforation group. The sigmoid shape of these curves is similar to that of the probability of perforation curves obtained experimentally. These experimental curves permit to define for a system projectile/laminate the ballistic limit $V_{50}$, that is, the impact velocity having a $50 \%$ of probability of perforation.

One can see in Fig. 8 how the width of the uncertainty band $\Delta v_{\mathrm{U}}$ of these curves, defined as the impact velocity range where $0<$ "PERFORATION YES" $<1$, increases with the impact angle. Table 6 shows the values of the uncertainty band for the different impact angles. This is consistent with the increase in the slope of the $V_{\mathrm{i}} \quad V_{\mathrm{r}}$ curves at the ballistic limit observed at high obliquities (Fig. 5a). A sharp increase in $V_{\mathrm{r}}$ with $V_{\mathrm{i}}$ would lead to a higher dispersion of the experimental results close to the bal listic limit.

Fig. 9 shows the output of MLP2: residual velocity and damaged area versus the impact velocity for five impact angles, some of them $\left(15^{\circ}, 40^{\circ}, 50^{\circ}\right)$ different from those employed in the training of the MLP. Fig. 9a shows how the damaged area is maximum for an impact angle of $0^{\circ}$ and how it increases with the angle at higher impact velocities. This result is also consistent with the experimen tal observations [25]. Fig. 9b shows how the ballistic limit in creases with the impact angle. Additionally the values obtained for this parameter with MLP2 are similar to those obtained with MLP1 (Fig. 8). At higher velocities, the curves tend to merge and the influence of the impact angle is negligible, as observed in experimental and numerical results.

Table 6

Width of the uncertainty band $\left(\Delta v_{\mathrm{U}}\right)$ as a function of the impact angle $\theta$

\begin{tabular}{llllll}
\hline$\theta$ & $0^{\circ}$ & $15^{\circ}$ & $30^{\circ}$ & $40^{\circ}$ & $50^{\circ}$ \\
\hline$\Delta v_{\mathrm{U}}^{\theta}(\mathrm{m} / \mathrm{s})$ & 44 & 44 & 52 & 60 & 72
\end{tabular}

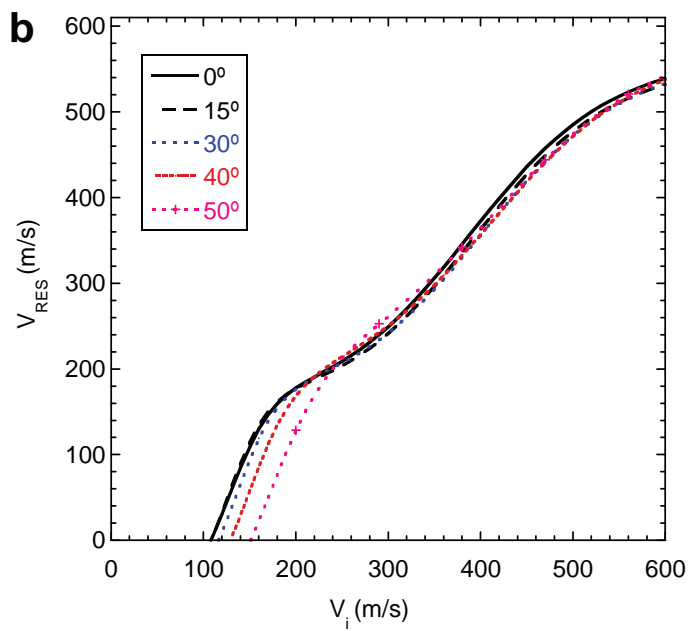

Fig. 9. Values predicted by the network: (a) damaged area vs. impact velocity for five impact angles; and (b) residual velocity vs. impact velocity for five impact angles. 


\section{Conclusions}

As a summary of the work, the following main conclusions can be drawn:

- The application of MLPs is effective in the prediction of occur rence of perforation and damaged area of the laminate, residual velocity and residual angle of the projectile. Accurate results have been obtained in the testing phase. Moreover, in the case examined, a simple network topology was able to give satisfac tory results.

- The network has shown a remarkable interpolation ability, pre dicting reasonable results for impact angles different from those used to train the neural network.

- The value of the parameter ballistic limit, of great interest for the study of the behaviour of systems against impact of solids, may be obtained by different network architectures and using differ ent input data, and similar results are obtained in both cases.

We conclude that the MLP may be considered as an alternative to the traditional methods to design CFRP laminates against high velocity impact of solids. The real time operation and the low cost of the MLP make it specially interesting during the first steps of the design, when is necessary to evaluate many different configura tions in the minimum time. Further development have to be done to include other input parameters such as laminate thickness and material properties.

\section{References}

[1] López-Puente J, Zaera R, Navarro C. The effect of low temperatures on the intermediate and high velocity impact response of CFRPs. Compos Part B: Eng 2002;33:559-66.

[2] Will M, Franz T, Nurick G. The effect of laminate stacking sequence of cfrp filament wound tubes subjected to projectile impact. Compos Struct 2002;58:259-70

[3] Hosur M, Vaidya U, Ulven C, Jeelani S. Performance of stitched/unstitched woven carbon/epoxy composites under high velocity impact loading. Compos Struct 2004;64:455-66.

[4] López-Puente J, Zaera R, Navarro C. High energy impact on woven laminates. J Phys IV 2003;110:639-44.

[5] Duan Y, Keefe M, Bogetti T, Cheeseman B. Modeling the role of friction during ballistic impact of a high-strength plain-weave fabric. Compos Struct $2005: 68: 331-7$.
[6] Chan S, Fawaz Z, Behdinan AR. Ballistic limit prediction using a numerical model with progressive damage capability. Compos Struct 2007:77:466-74.

[7] Morye S, Hine P, Duckett R, Carr D, Ward I. Modelling of the energy absorption by polymer composites upon ballistic impact. Compos Sci Technol 2000;60:2631-42.

[8] Bohong G. Analytical modeling for the ballistic perforation of planar plainwoven fabric target by projectile. Compos Part B: Eng 2003;34:361-71.

[9] Caprino G, Lopresto V, Santoro D. Ballistic impact behaviour of stitched graphite/epoxy laminates. Compos Sci Technol 2007;67(3-4):325-35.

[10] López-Puente J, Zaera R, Navarro C. An analytical model for high velocity impacts on thin CFRPs woven laminated plates. Int J Solid Struct 2007;44:2837-51.

[11] Xu Y, Liu G, Wu Z, Huang X. Adaptive multilayer perceptron networks for detection of cracks in anisotropic laminated plates. Int J Solid Struct 2001;38:5625-45.

[12] Just-Agosto F, Serrano D, Cecchini SBA. Neural network based nondestructive evaluation of sandwich composites. Compos Part B: Eng 2008;39(1):217-25.

[13] Kesavan A, Deivasigamani M, John S, Herszberg I. Damage detection in t-joint composite structures. Compos Struct 2006;75(1-4):313-20.

[14] Yam L, Yan Y, Jiang J. Vibration-based damage detection for composite structures using wavelet transform and neural network identification. Compos Struct 2003;60(4):403-12.

[15] Seo D, Lee J. Damage detection of cfrp laminates using electrical resistance measurement and neural network. Compos Struct 1999;47(1-4):525-30.

[16] Mahdi E, El Kadi H. Crushing behavior of laterally compressed composite elliptical tubes: experiments and predictions using artificial neural networks. Compos Struct 2008;83:399-412.

[17] El Kadi H. Modeling the mechanical behavior of fiber-reinforced polymeric composite materials using artificial neural networks a review. Compos Struct 2006;73(1):1-23.

[18] Chandrashekhara K, Okafor A, Jiang Y. Estimation of contact force on composite plates using impact-induced strain and neural networks. Compos Part B: Eng 1998;29(4):363-70.

[19] Fernández-Fdz D, Zaera R. A new tool based on artificial neural networks for the design of lightweight ceramic-metal armors against high velocity impact of solids. Int J Solid Struct.

[20] Tiago C, Leitão V. Application of radial basis functions to linear and nonlinear structural analysis problems. Compos Math Appl 2006;51(8):1311-34.

[21] Deng J. Structural reliability analysis for implicit performance function using radial basis function network. Int J Solid Struct 2006;43(11-12):3255-91.

[22] Príncipe JC, Neil RE, Lefebvre C. Neural and adaptive systems: fundamentals through simulations. NY: John Wiley; 1999 [Chapter 4].

[23] HKS. Abaqus Explicit v6.4 User's Manual, version 6.4 Edition, ABAQUS Inc., Richmond, USA; 2003.

[24] Hou JP, Petrinic N, Ruiz C, Hallet SR. Prediction of impact damage in composite plates. Compos Sci Technol 2000;60:273-81.

[25] López-Puente J, Zaera R, Navarro C. Experimental and numerical analysis of normal and oblique ballistic impacts on thin carbon/epoxy woven laminates. Compos Part A-Appl Sci 2007;39:374-87.

[26] NDS. NeuroSolutions for Excel v4.21 User's Manual, version 4.21 Edition, NeuroDimension Inc., Gainesville, USA; 2003.

[27] Tarassenko L. A guide to neural computing applications. London: Arnold/ NCAF; 1998 